\title{
Real-time management of berth allocation with stochastic arrival and handling times
}

\author{
Nitish Umang * $\quad$ Michel Bierlaire * Alan L. Erera ${ }^{\dagger}$
}

Report TRANSP-OR 130215

Transport and Mobility Laboratory

Ecole Polytechnique Fédérale de Lausanne

transp-or.epfl.ch

\footnotetext{
${ }^{*}$ Transport and Mobility Laboratory (TRANSP-OR), School of Architecture, Civil and Environmental Engineering (ENAC), Ecole Polytechnique Fédérale de Lausanne (EPFL), CH-1015 Lausanne, Switzerland, \{nitish.umang, michel.bierlaire\}@epfl.ch

†School of Industrial and Systems Engineering, Georgia Institute of Technology, Atlanta, Georgia 30332, USA, alerera@isye.gatech.edu
} 


\begin{abstract}
In this research we study the berth allocation problem (BAP) in real time as disruptions occur. In practice, the actual arrival times and handling times of the vessels deviate from their expected or estimated values, which can disrupt the original berthing plan and potentially make it infeasible. We consider a given baseline berthing schedule, and solve the BAP on a rolling planning horizon with the objective to minimize the total realized costs of the updated berthing schedule as the actual arrival and handling time data is revealed in real time. The uncertainty in the data is modeled by making appropriate assumptions about the probability distributions of the uncertain parameters based on past data. We present an optimization based recovery algorithm based on set partitioning method and a smart greedy algorithm to reassign the vessels in the events of disruption. Our research problem derives from the real world issues faced by the SAQR port, Ras Al Khaimah, UAE, where the berthing plans are regularly disrupted owing to a high degree of uncertainty in information. A simulation study is carried out to assess the solution performance and efficiency of the proposed algorithms, in which the baseline schedule is chosen as the solution of the deterministic berth allocation problem without accounting for any uncertainty. Results indicate that the proposed algorithms can significantly reduce the total realized costs of the berthing schedule as compared to the ongoing practice of reassigning vessels at the port.
\end{abstract}




\section{Introduction}

The Berth Allocation Problem (BAP) is one of the most critical and widely studied problems in seaport operations planning. Port operations are affected by a high degree of vessel travel time and handling time uncertainty arising from weather conditions, mechanical problems, port congestion, demand uncertainty, and other factors. Such uncertainty can make berth allocation planning difficult, and planned schedules are often disrupted. To minimize the impact of such disruptions, plans must be updated dynamically. Most optimization-based approaches for creating berth allocation plans do not explicitly account for uncertainty. Furthermore, the objectives used in such approaches do not explicitly consider objectives useful during re-planning.

Two approaches are used for managing uncertain disruptions in transportation scheduling. In the first approach, systematic robustness is built into the planned, or baseline, schedule. Stochastic optimization models (see Birge and Louveaux (1997), Kall and Mayer (2005) and Wallace and Ziemba (1997)) address problems of this type by minimizing expected operational costs given a probabilistic representation of possible outcomes, while robust optimization models (see Soyster (1973), Bertsimas and Sim (2003), Ben-Tal and Nemirovski (1998), Ben-Tal and Nemirovski (1999), Ben-Tal and Nemirovski (2000) and Bertsimas and Sim (2004)) alternatively focus on worst-case performance over some subset of possible outcomes. The second approach to managing disruptions is to build reactive models for modifying a schedule in real-time in response to new information; it is common to refer to these optimization problems as recovery problems. Recovery optimization problems usually use a deterministic information model. To measure the effectiveness of dynamic reactive models, a competitive ratio between the system cost resulting from repeated application of a reactive optimization model and the optimal cost found by a posteriori optimization may be computed (Albers (2003)).

In this paper, we consider the problem of real-time berth rescheduling. The underlying model is the dynamic, hybrid berth allocation model for bulk ports developed by Umang et al. (2013). We consider uncertainty in both the arrival times and the handling times of the vessels. The objective is to minimize the total realized costs of the modified berthing schedule, which is the sum of the total service cost of the vessels, the rescheduling costs created by altering the berthing times and positions of vessels from a baseline schedule, and the delay to arriving vessels, discussed in more detail later in the paper.

\section{Literature Review}

Comprehensive literature surveys covering operations research approaches to berth allocation problems in container terminals can be found in Bierwirth and Meisel (2010), Steenken et al. (2004) and Stahlbock and Voss (2008). The deterministic berth allocation problem (BAP) in bulk ports with dynamic vessel arrivals and hybrid berth layout is studied by Umang et al. (2013).

Few studies propose robust planning methods for berth allocation, although are some recent examples. Zhen et al. (2011) use a meta-heuristic approach to solve a two-stage stochastic BAP given a fixed set of scenarios, where the objective is to minimize the total cost of a baseline schedule and the expected cost of recourse. The recourse cost in this study is the weighted time and space deviation of the realistic schedule from the baseline schedule. Han et al. (2010) use a simulation-based genetic 
algorithm to solve an integrated berth and quay crane scheduling problem with uncertainty in vessel arrival and operation times. For given probability density functions, the objective is to minimize the sum of expected value and standard deviation of the service time and the weighted tardiness of the vessels.

Other papers propose surrogate measures of berth schedule robustness, and incorporate these measures into an optimization objective. For example, Moorthy and Teo (2006) use a sequence pair approach to design a robust berth template for transshipment hubs in container terminals, in which the conflicting objectives are to minimize the total expected delays and deviation from the most preferred berthing locations. Zhen and Chang (2012) define robustness as the weighted sum of the free slack times in the berthing schedule, where weights are determined according to the vessel priorities. A bi-objective model is proposed that minimizes cost and maximizes robustness. Xu et al. (2012) solve a continuous berth allocation problem with uncertainty in vessel arrival and handling times, in which the objective is to balance level of service measured by total vessel departure delay with a robustness measure defined by length of time buffers inserted between vessels occupying the same berthing location to absorb uncertain delays.

Little research has addressed real-time management of berth allocation. In practice, simple rules of thumb guide rescheduling of vessels. Since actual vessel arrival times, and to a lesser extent vessel handling times, may differ substantially from those assumed when developing a baseline schedule, it should be clear that rescheduling will often be required and it is important to do so effectively. A simple but naive approach to rescheduling is to not shift planned vessel berthing positions when rescheduling, and to simply serve vessels at the earliest feasible time given a first-in first-out (FIFO) ordering specified by the planned berthing times. A different approach is to apply an optimization model in a roll-out procedure for vessel rescheduling, but doing so requires some attention to detail. Zeng et al. (2012) and Du et al. (2010) are a couple of examples of related works. Zeng et al. (2012) address the problem of disruption recovery in the integrated berth and quay crane assignment problem in container terminals. They develop optimization models for re-allocation of berth assignment and rescheduling of quay cranes, but solve the disruption recovery problem using local rescheduling and tabu search methods. Du et al. (2010) use a feedback procedure to develop a robust berth allocation plan and a reactive strategy that takes into account the priorities assigned to the vessels and the congestion at the port.

In this research, we develop a methodology to model the uncertainty in the yet-to-be-revealed arrival times and handling times of the vessels, based on probability distributions derived from past data. We propose a recovery algorithm based on re-optimization of the berthing schedule in the events of disruption and a heuristic based smart greedy algorithm for berth rescheduling in real time. The objective is to minimize the total realized cost of the updated schedule. Our research problem is motivated by challenges faced along these lines at the SAQR port, Ras al-Khaimah, United Arab Emirates, where planned operations are frequently disrupted due to a high degree of uncertainty in the vessel arrival and handling times. 


\section{Problem Statement}

\subsection{Baseline Schedule}

We study the berth allocation problem in real time for a given baseline schedule. The vessel arrival process is dynamic and stochastic. We assume a hybrid berthing layout and a fixed planning horizon partitioned into discrete time buckets, where each vessel may occupy multiple discrete berth sections, but a given section may be occupied by at most one vessel at a given time, as shown in Figure 1 .

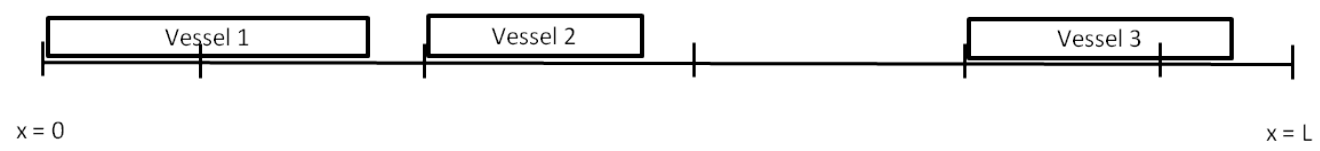

Figure 1: Hybrid berthing layout, showing a feasible assignment of vessels to berth sections at a single point in time

Umang et al. (2013) demonstrates that the dynamic hybrid berth allocation problem with known arrival and handling times can be effectively modeled and solved as a generalized set-partitioning problem (GSPP) for relatively large problem size and time horizon of few days. In this approach, the set of all feasible single-vessel berthing assignments is generated a priori and is denoted by the set $P$. Note that a berthing assignment for a single vessel specifies the berth sections that will be occupied by the vessel, its berthing time, and its completion time (equal to the berthing time plus the handling time). The assignment matrix contains a column for each of the $|\mathrm{P}|$ assignments, and is composed of upper submatrix $A$ and lower submatrix $B$. Each column $p$ in submatrix $A$ has a single non-zero value, where row $i$ contains the value one if the berthing assignment is for vessel $i \in N$. Submatrix $B$ contains a single row for each (berth section, time bucket). Non-zero values in submatrix B are equal to one if the vessel berthing assignment specified by column $p$ requires that the vessel occupies the (section, time) represented by the row. To illustrate this idea, consider an example with two vessels 1 and 2 as shown in Figure 2. Suppose the quay has 3 discrete berth sections, and that the planning horizon has 3 discrete time periods. Vessel 1 requires specialized handling equipment only available in sections 1 and 2, and vessel 2 arrives at the start of time 2. The resulting assignment matrix is shown in Table 1. The first column represents the berthing assignment of vessel 1 to sections 1 and 2 from time 1-2, and so on.

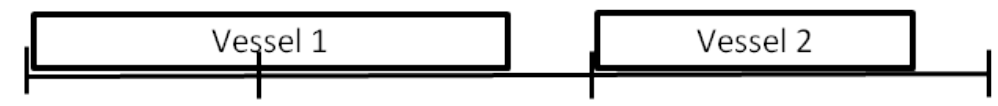

$$
\mathrm{x}=0 \quad \mathrm{x}=\mathrm{L}
$$

Figure 2: Simple example of set partitioning to solve the $B A P$ with $|\mathrm{N}|=2,|\mathrm{M}|=3$ and $|\mathrm{H}|=3$

We assume the following input data to be available for the GSPP model: 


\begin{tabular}{|c|c|c|c|c|}
\hline Vessel 1 & 1 & 1 & 0 & 0 \\
Vessel 2 & 0 & 0 & 1 & 1 \\
Section 1, Time 1 & 1 & 0 & 0 & 0 \\
Section 1, Time 2 & 1 & 1 & 1 & 0 \\
Section 1, Time 3 & 0 & 1 & 1 & 0 \\
Section 2, Time 1 & 1 & 0 & 0 & 0 \\
Section 2, Time 2 & 1 & 1 & 1 & 1 \\
Section 2, Time 3 & 0 & 1 & 1 & 1 \\
Section 3, Time 1 & 0 & 0 & 0 & 0 \\
Section 3, Time 2 & 0 & 0 & 0 & 1 \\
Section 3, Time 3 & 0 & 0 & 0 & 1 \\
\hline
\end{tabular}

Table 1: Assignment matrix for a simple example of GSPP

$\mathrm{H}=$ set of discrete time intervals in the planning horizon

$\mathrm{P}=$ set of feasible assignments

$\mathrm{s}=1, \ldots,|\mathrm{H}| \quad$ discrete time intervals in the planning horizon

$p=1, \ldots,|P| \quad$ feasible assignments

$d_{p}=$ delay associated with assignment $p$

$h_{p}=$ handling time associated with assignment $p$

The assignment matrix coefficients are defined as follows.

$A_{i p}= \begin{cases}1 & \text { if vessel } i \text { is the assigned vessel in the feasible assignment represented } \\ & \text { by assignment } p \\ 0 & \text { otherwise. }\end{cases}$

$b_{p}^{k s}= \begin{cases}1 & \text { if section } k \text { is occupied at time } s \text { in assignment } p \\ 0 & \text { otherwise. }\end{cases}$

There is only a single decision variable in the GSPP model for selection of feasible assignments in the optimal solution which is defined as follows.

$\lambda_{p}= \begin{cases}1 & \text { if assignment } p \text { is part of the optimal solution; } \\ 0 & \text { otherwise. }\end{cases}$

The GSPP model is formulated as shown below: 


$$
\begin{aligned}
& \min \sum_{p}\left(d_{p} \lambda_{p}+h_{p} \lambda_{p}\right) \\
& \text { s.t. } \sum_{p}\left(A_{i p} \lambda_{p}\right)=1 \quad \forall i \in N \\
& \sum_{p}\left(b_{p}^{k s} \lambda_{p}\right) \leq 1 \quad \forall k \in M, \forall s \in H \\
& \lambda_{p} \in\{0,1\} \quad \forall p \in P
\end{aligned}
$$

In the deterministic model, the objective (1) is to minimize the total service cost of the vessels berthing at the port, which includes the total berthing delays and the total handling cost of the vessels. Constraints (2) ensure that each vessel must have exactly one feasible assignment in the optimal solution. Constraints (3) ensure that a given section at a given time can be occupied by at most one vessel.

\subsection{Real Time Recovery}

In practice, the actual arrival and handling times of vessels may deviate from their estimated values, which can disrupt the baseline schedule and possibly render it infeasible. To create a model for berth schedule recovery, we first describe a dynamic information model based on actual seaport operations. We assume that the port receives dynamic updates on the estimated arrival time of each inbound vessel. Suppose that these estimated arrival time updates occur sporadically for each vessel, and that each update occurs before the actual arrival of the vessel at time $a_{i}$. We assume that $a_{i}$ is known with certainty only at time $a_{i}$. In the case of handling time, we assume that a single estimate of handling time is known in advance and that actual handling time $h_{i}$ of vessel $i$ is only known when handling is completed.

To design an optimization problem for schedule recovery, suppose that re-planning is initiated at time $t$ for a given baseline schedule. At this time, we can partition the vessel set $\mathrm{N}$ into five subsets as follows:

$$
N=N_{1}^{t} \cup N_{2}^{t} \cup N_{3}^{t} \cup N_{4}^{t} \cup N_{5}^{t}
$$

where,

- $\mathrm{N}_{1}^{\mathrm{t}}$ is the subset of vessels which have been berthed, completely served, and have departed the port;

- $\mathrm{N}_{2}^{\mathrm{t}}$ is the subset of vessels which are currently berthed, at known berthing locations that cannot be altered;

- $\mathrm{N}_{3}^{\mathrm{t}}$ is the subset of vessels which arrived to the port, but have not yet been berthed; 


\begin{tabular}{|c|c|c|c|}
\hline Vessel & Expected Arrival Time & Baseline Berthing Time & Expected Handling Time \\
\hline 1 & 0 & 0 & 3 \\
2 & 0 & 1 & 2 \\
3 & 0 & 1 & 2 \\
\hline
\end{tabular}

Table 2: Baseline schedule for illustrative example with $|\mathrm{N}|=3,|\mathrm{M}|=6$ and $|\mathrm{H}|=4$

- $\mathrm{N}_{4}^{\mathrm{t}}$ is the subset of vessels which have not arrived yet, but who have an estimated arrival time $\hat{a}_{i}^{t}>t ;$ and

- $\mathrm{N}_{5}^{\mathrm{t}}$ is the subset of vessels which have not arrived yet, but who have an estimated arrival time $\hat{a}_{i}^{t} \leq t$.

Note that the set of unassigned vessels at time instant $t$ is $N_{u}^{t}=N_{3}^{t} \cup N_{4}^{t} \cup N_{5}^{t}$.

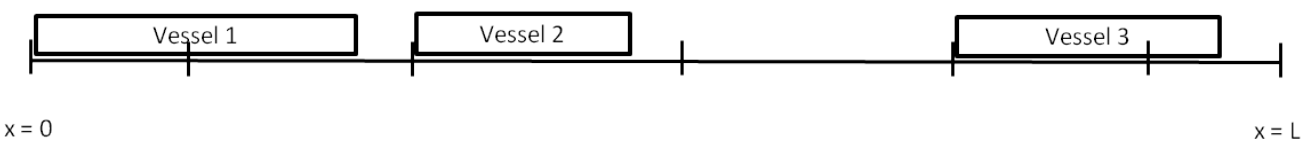

Figure 3: Baseline berthing positions for illustrative example with $|\mathrm{N}|=3,|\mathrm{M}|=6$ and $|\mathrm{H}|=4$

Consider the example containing three vessels 1,2 and 3 as shown in Figure (3). The berthing positions of the vessels in the original baseline schedule are as shown in the figure. We consider 6 quay sections and 4 discrete time intervals in the planning horizon. The arrival and handling time information related to all the vessels is given in Table 2

Now consider the disruption scenario detailed in Table 3. Each large row in the table describes the events that have occurred by time $t$. Note that the arrival time of vessel 1 is updated twice, and it actually arrives at time 2 . Although the expected handling time for vessel 1 is 3 , actual handling time is 2 and the vessel departs at time 4 . The arrival time of vessel 2 is updated once, and the actual handling time is equal to the expected value of 2 . Note that at time $t=1$, vessel 2 belongs to the subset $\mathrm{N}_{5}^{\mathrm{t}}$, since there is no available information about the future expected arrival time of the vessel. For vessel 3, the actual arrival and handling times are the same as the expected values and there are no arrival information updates.

Once a berthing schedule is determined, the terminal manager may begin allocating resources such as cargo storage facilities, labour, and handling equipment according to the requirements of the berthing vessels. When the baseline schedule is disrupted, the manager must reallocate these resources and incur incur additional costs. Thus, an important objective in berthing schedule recovery is to minimize the deviation of the realized berthing plan from the baseline. Another important measure is the fairness of any required rescheduling. Here, we attempt to ensure fairness by prioritizing the minimization of berthing delay for vessels that arrive on time. Finally, it is also important to maximize overall port productivity which we measure using the total actual flow time of all berthing vessels in the usual way. 


\begin{tabular}{|c|c|c|c|c|c|c|}
\hline Time & Vessel & Arrival Time Updated? & Vessel Arrived & Vessel Assigned & Vessel Completed & Subset \\
\hline \multirow{3}{*}{$\mathrm{t}=0$} & 1 & Yes $\rightarrow 1$ & No & No & No & $\mathrm{N}_{4}^{\mathrm{t}}$ \\
\hline & 2 & Yes $\rightarrow 1$ & No & No & No & $\mathrm{N}_{4}^{\mathrm{t}}$ \\
\hline & 3 & - & Yes & No & No & $\mathrm{N}_{3}^{t}$ \\
\hline \multirow{3}{*}{$\mathrm{t}=1$} & 1 & Yes $\rightarrow 2$ & No & No & No & $\mathrm{N}_{4}^{\mathrm{t}}$ \\
\hline & 2 & No & No & No & No & $\mathrm{N}_{5}^{t}$ \\
\hline & 3 & - & Yes & Yes & No & $\mathrm{N}_{2}^{t}$ \\
\hline \multirow{3}{*}{$t=2$} & 1 & - & Yes & Yes & No & $\mathrm{N}_{2}^{t}$ \\
\hline & 2 & - & Yes & Yes & No & $\mathrm{N}_{2}^{\mathrm{t}}$ \\
\hline & 3 & - & Yes & Yes & No & $\mathrm{N}_{2}^{\mathrm{t}}$ \\
\hline \multirow{3}{*}{$t=3$} & 1 & - & Yes & Yes & No & $\mathrm{N}_{2}^{\mathrm{t}}$ \\
\hline & 2 & - & Yes & Yes & No & $\mathrm{N}_{2}^{\mathrm{t}}$ \\
\hline & 3 & - & Yes & Yes & Yes & $\mathrm{N}_{1}^{\mathrm{t}}$ \\
\hline \multirow{3}{*}{$t=4$} & 1 & - & Yes & Yes & Yes & $\mathrm{N}_{1}^{\mathrm{t}}$ \\
\hline & 2 & - & Yes & Yes & Yes & $\mathrm{N}_{1}^{\mathrm{t}}$ \\
\hline & 3 & - & Yes & Yes & Yes & $\mathrm{N}_{1}^{\mathrm{t}}$ \\
\hline
\end{tabular}

Table 3: Disruption Scenario for a simple example with $|N|=3,|M|=6$ and $|H|=4$

We propose therefore the following optimization model for real time recovery of a baseline berth schedule. The model is to be solved given a baseline schedule at time epoch $t$, and considers only a single scenario for all uncertain parameters (to be described in more detail in Section 4.1). 
The input parameters for the model are:

$|\mathrm{H}|=$ duration of planning horizon

$\mathrm{L}=$ total length of quay

$\mathrm{M}=$ set of berth sections

$\mathrm{N}_{\mathrm{a}}^{\mathrm{t}}=$ subset of active vessels at time $\mathrm{t}$ that have not yet departed the port

$N_{\mathfrak{u}}^{t}=$ subset of vessels at time $t$ that have not been assigned to a berth position

$\mathrm{N}_{\mathrm{o}}^{\mathrm{t}}=$ subset of unassigned vessels at time $t$ that arrived on-time or are expected to arrive on-time

$A_{i}=$ planned arrival time of vessel $i$

$\mu_{i}=$ service priority of vessel $i$

$a_{i}^{t}=$ (updated) arrival time of vessel $i$ at time $t$

$b_{i k}=1$ if baseline berthing location $k \in M$ used for vessel $i$ (starting berth section), 0 otherwise

$m_{i}=$ baseline berthing time of vessel $i$

$e_{i}=$ baseline departure time of vessel $i$

$g_{k}=$ linear coordinate of berthing location $k$

$\mathrm{L}_{\mathrm{i}}=$ length of vessel $i$

$h_{i k}^{t}=$ (updated) handling time at time $t$ for vessel $i$ berthed at starting berth section $\mathrm{k}$

$M_{i} \subseteq M$, subset of starting berth sections for which vessel can be feasibly berthed due to draft and length restrictions

$c_{1}=\quad$ penalty cost of shifting a vessel by a unit distance along the quay

$c_{2}=$ penalty cost of unit delay time beyond baseline departure time for a vessel

$c_{3}=$ penalty cost of unit delay time beyond baseline service time for a vessel arriving on-time

The decision variables are:

$m_{i}^{\prime} \geq 0 \quad$ updated berthing time of vessel $i$

$e_{i}^{\prime} \geq 0 \quad$ updated departure time of the vessel $i$

$w_{i}^{\prime} \geq 0$ difference between the updated service time and the estimated service time as per the baseline schedule of the vessel $i$

$b_{i k}^{\prime} \quad$ binary, equals 1 if vessel $i$ updated berthing location is $k, 0$ otherwise

$y_{i j} \quad$ binary, equals 1 if vessel $i$ berthed to the left of vessel $j$ along quay, 0 otherwise

$z_{i j} \quad$ binary, equals 1 if vessel $i$ departs no later than the berthing time of vessel $j, 0$ otherwise

The optimization model can be formulated as follows: 


$$
\begin{aligned}
& \min Z_{t}=Z_{1 \mathrm{t}}+Z_{2 \mathrm{t}}+Z_{3 \mathrm{t}} \\
& Z_{1 t}=\sum_{i \in N_{u}^{t}}\left(e_{i}^{\prime}-a_{i}^{t}\right) \\
& \mathrm{Z}_{2 \mathrm{t}}=\sum_{i \in \mathrm{N}_{\mathfrak{u}}^{\mathrm{t}}}\left(c_{1}\left|\sum_{k \in M} g_{k} b_{i k}^{\prime}-\sum_{k \in M} g_{k} b_{i k}\right|+c_{2} \mu_{i}\left|e_{i}^{\prime}-e_{i}\right|\right) \\
& \mathrm{Z}_{3 \mathrm{t}}=\sum_{i \in \mathrm{N}_{\mathrm{o}}^{t}} \mathrm{c}_{3} w_{\mathrm{i}}^{\prime}
\end{aligned}
$$

subject to the constraints

$$
\begin{aligned}
& m_{i}^{\prime}-a_{i}^{t} \geq 0 \quad \forall i \in N_{u}^{t} \\
& e_{i}^{\prime}-m_{i}^{\prime}-\sum_{k \in M} h_{i k}^{t} b_{i k}^{\prime}=0 \quad \forall \quad i \in N_{a}^{t} \\
& w_{i}^{\prime} \geq\left(e_{i}^{\prime}-a_{i}^{t}\right)-\left(e_{i}-A_{i}\right) \quad \forall \quad i \in N_{o}^{t} \\
& \sum_{k \in M}\left(g_{k} b_{j k}^{\prime}\right)+B\left(1-y_{i j}\right) \geq \sum_{k \in M}\left(g_{k} b_{i k}^{\prime}\right)+L_{i} \quad \forall \quad i \in N_{a}^{t}, j \in N_{u}^{t}, i \neq j \\
& m_{j}^{\prime}+B\left(1-z_{i j}\right) \geq m_{i}^{\prime}+\sum_{k \in M} h_{i k}^{t} b_{i k}^{\prime} \quad \forall \quad i \in N_{a}^{t}, j \in N_{u}^{t}, i \neq j \\
& y_{i j}+y_{j i}+z_{i j}+z_{j i} \geq 1 \quad \forall \quad i \in N_{a}^{t}, j \in N_{u}^{t}, i \neq j \\
& \sum_{k \in M_{i}} b_{i k}^{\prime}=1 \quad \forall i \in N_{u}^{t} \\
& b_{i k}^{\prime} \in\{0,1\} \quad \forall \quad i \in N_{a}^{t}, \forall k \in M \\
& y_{i j} \in\{0,1\} \quad \forall \quad i \in N_{a}^{t}, j \in N_{u}^{t} \\
& z_{i j} \in\{0,1\} \quad \forall \quad i \in N_{a}^{t}, j \in N_{u}^{t}
\end{aligned}
$$

The equations (5)-(8) minimize the total realized cost $Z_{t}$ at time instant $t$, which is the sum of the total service cost of the vessels given by $Z_{1 t}$, the total cost of rescheduling the vessels given by $Z_{2 t}$ and the delays beyond the estimated service times of vessels arriving on-time given by $Z_{3 t}$. Note that while the parameters $\mu_{i}$ are dependent on the relative priorities assigned by the port authority to the vessels berthing at the port, the choice of the parameters $c_{1}, c_{2}$ and $c_{3}$ needs to be adapted in accordance with the actual real cost incurred by the port due to each of the cost components under different disruption scenarios. In the absence of such data, the parameter values were chosen on the basis of intuition and trials in our study. Constraints (9) are the dynamic arrival constraints. Constraints (10) state that vessels depart as soon as their processing is finished. Note that in constraints $(10), m_{i}^{\prime}$ and $b_{i k}^{\prime}$ are preset and unchangeable for vessels that have already been assigned to a berth position. 
Constraints (11) are used to define the variables $w_{i}^{\prime}$ as the difference in the updated service time and the estimated service time of the vessel $i$ as per the baseline schedule. Constraints (12)-(14) are the non-overlapping restrictions for any two vessels berthing at the port. Constraints (15) ensure that each vessel has exactly one starting berth section, and occupies sections for which the vessel can be feasibly berthed due to length and draft restrictions. Note that the vessel arrival times and handling times in the objective function term (6) and the constraints $(9),(10),(11)$ and (13) do not represent the actual values, but our expectation of the arrival and handling time values at time instant $t$, which may or may not have been revealed up to that time instant. The modeling of uncertainty in the arrival times and handling times of the vessels is discussed in the next section.

\section{Recovery Methodology}

\subsection{Modeling Uncertainty}

We propose that recovery decisions to be determined via optimization over a rolling planning horizon. At any given time $t$, certain vessel arrival and handling times are known with certainty, while other information is not known.

\subsubsection{Uncertainty in arrival times}

We use a simple approach for modeling uncertain arrival times. Based on sample data from the port, we assume that vessel arrival times are uniformly distributed around the expected arrival time. Specifically, for vessel $i \in N$, the actual arrival time $a_{i}$ lies in the interval $\left[A_{i}-V, A_{i}+V\right]$ where $A_{i}$ is the expected arrival time. When planning, we assume that the most recent update $a_{i}^{t}$ is appropriate for use during planning. Then, we model arrival times at time $t$ as follows:

- If vessel $i \in N_{2}^{t} \cup N_{3}^{t}$, implying that the vessel has actually arrived, then $a_{i}^{t}$ is known and equal to $a_{i}$;

- If vessel $i \in N_{4}^{t}$, then $a_{i}^{t}$ is assumed equal to the last update of its arrival time. Thus if the updated arrival time of the vessel at time $t_{1} \leq t$ was $a_{i}^{t_{1}}$ and no other arrival time update occurs between $t_{1}$ and $t$, then the planned arrival time $a_{i}^{t}$ is assumed equal to $a_{i}^{t_{1}}$; and

- If vessel $i \in \mathrm{N}_{5}^{\mathrm{t}}$, then the most recent vessel arrival time update was inaccurate and we therefore assume that the vessel may arrive any time on the interval $\left[t, A_{i}+V\right]$. We use a planning time $a_{i}^{t}$ where

$$
\operatorname{Prob}\left(a_{i} \leq a_{i}^{t}\right)=\rho_{a}
$$

where $\rho_{a}$ is an input probability and $a_{i}$ is assumed to be uniformly distributed on $\left[t, A_{i}+V\right]$, and thus 


$$
a_{i}^{t}=t+\rho_{a}\left(A_{i}+V-t\right)
$$

Note that $1-\rho_{a}$ can be interpreted as a likelihood of the infeasibility of the schedule determined at time instant $t$ due to late arrival of vessels in $\mathrm{N}_{5}^{\mathrm{t}}$.

\subsubsection{Uncertainty in handling times}

Based on sample data from the port, the handling times of vessels are modeled using truncated exponential distributions. In practice, actual handling times are usually close to estimated values, but in cases of equipment breakdown or other mechanical problems the times may be significantly longer. For any vessel $i \in N$ berthed at the starting section $k \in M$, the handling time is assumed to be distributed according to a truncated exponential distribution on the interval $\left[\mathrm{H}_{i k}, \gamma \mathrm{H}_{i k}\right]$ where $\mathrm{H}_{i k}$ is the minimum handling time and $\gamma \geq 1$ is a factor used to define an upper bound. We then use the following two cases to determine a planned handling time $h_{i k}^{t}$ at time $t$ :

- If vessel $i \in \mathrm{N}_{2}^{\mathrm{t}}$, then its actual handling time is not yet known but its berth position $k$ is known. Let $g_{i t}$ be the elapsed processing time of vessel $i$ at time $t$. Then, the total handling time $h_{i k}$ is assumed to be distributed according to a truncated exponential distribution on the interval $\left[\max \left(g_{i t}, H_{i k}\right), \gamma H_{i k}\right]$, and $h_{i k}^{t}$ is determined such that

$$
\operatorname{Prob}\left(h_{i k} \leq h_{i k}^{t}\right)=\rho_{h}
$$

where $\rho_{h}$ is an input probability. Again, $1-\rho_{h}$ is the likelihood that the vessel handling time, realized at time $t$, exceeds $h_{i k}^{t}$ and thus potentially invalidates the replanned schedule.

- If vessel $i \in N_{3}^{t} \cup N_{4}^{t} \cup N_{5}^{t}$, then neither the actual handling time of the vessel nor the actual berthing position of the vessel are yet known. In this case, $h_{i k}$ is assumed to be distributed according to a truncated exponential distribution on the interval $\left[\mathrm{H}_{i k}, \gamma \mathrm{H}_{i k}\right]$, and $h_{i k}^{\mathrm{t}}$ is determined using the same expression

$$
\operatorname{Prob}\left(h_{i k} \leq h_{i k}^{t}\right)=\rho_{h}
$$

Note that in these cases, the expected handling time of vessel $i$ at time $t$ berthed at position $k$ is given by

$$
h_{i k}^{t}=-(1 / \tau) \ln \left(e^{-\tau L_{i k}^{t}}-\rho_{h}\left(e^{-\tau L_{i k}^{t}}-e^{-\tau U_{i k}^{t}}\right)\right)
$$

where $L_{i k}^{t}$ and $U_{i k}^{t}$ are the left and right extremes of the discrete truncated exponential distribution of the handling time of the vessel at time $t$, and $\tau$ is the parameter of the distribution. 


\subsection{Solution Algorithms}

\subsubsection{Traditional Greedy Algorithm}

In this section, we briefly discuss the current practice for reassigning vessels given a baseline schedule at the port. We call the corresponding algorithm the traditional greedy algorithm for schedule recovery. In this approach, we move forward in time from present and assign each incoming vessel to the berthing location where the cost of reassigning the vessel is minimized. The cost of reassignment includes all the three cost components associated with the particular vessel to be reassigned. A vessel is assigned to a berthing location as soon as space is available, but not before the planned berthing time in the original baseline schedule. The implementation is presented in detail in Algorithm 1 .

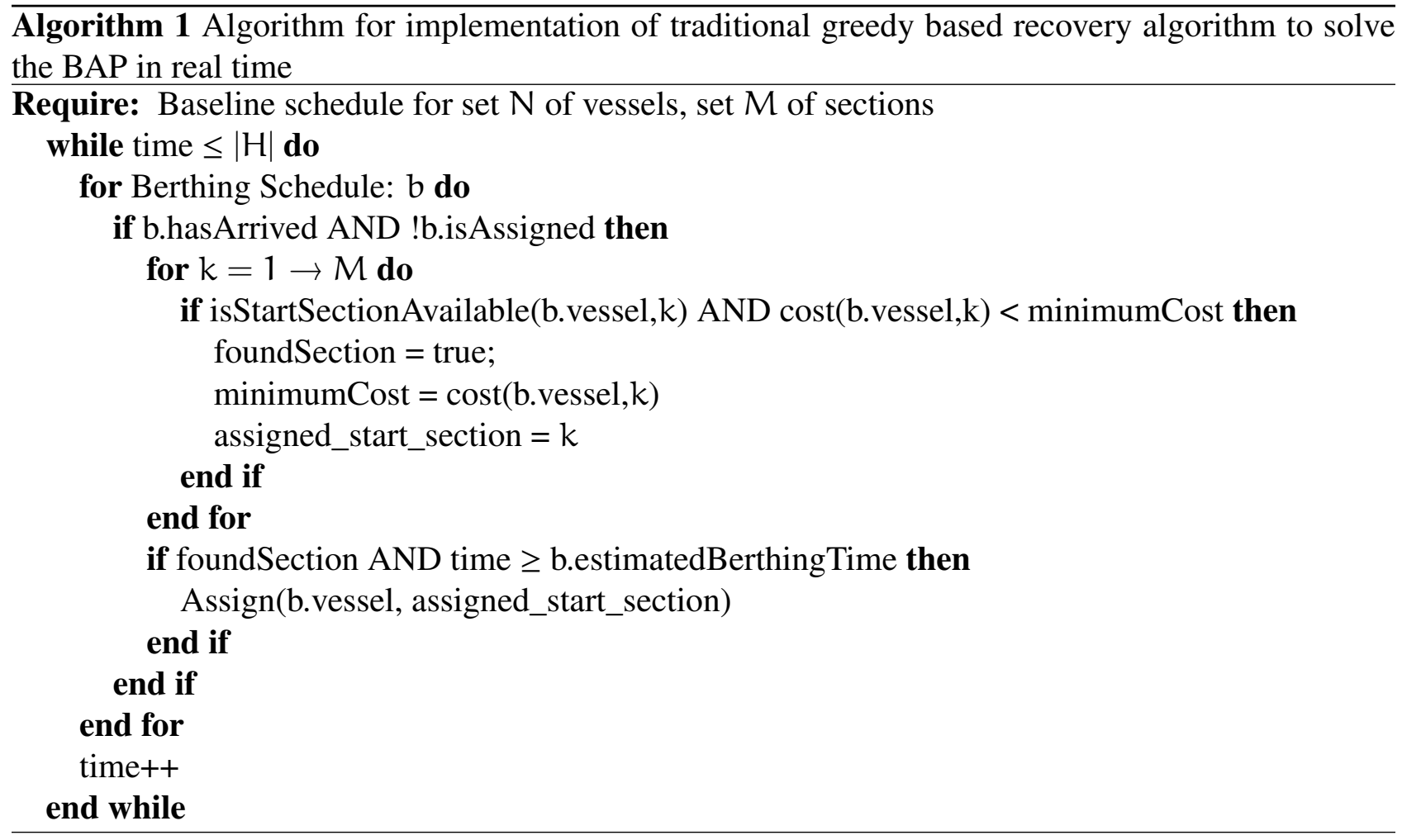

\subsubsection{Optimization Based Recovery Algorithm}

The proposed optimization methodology for schedule recovery in real time seeks to re-plan berthing assignments for all vessels not currently berthed at each decision epoch given new information. Replanning is only required when:

- the arrival time of any vessel is updated, and it deviates from its previous value

- the actual handling time of any vessel is revealed and is not equal to its estimated value 
In a given optimization run, the new berthing assignment is determined by the re-optimization of all the unassigned vessels in the schedule at that time instant with the objective function (19). The uncertainty in the yet-to-be-revealed arrival times and handling times of the vessels is modeled as described in section 4.1. When solving the problem at time instant $t$ for the rolling planning horizon $[t, t+H]$, the berthing assignment of all the vessels whose processing has already started is considered frozen and unchangeable. To prevent space overlapping with vessels which are being currently processed, the occupied sections are blocked for the worst handling time for each of the berthed vessels until their actual handling time is revealed.

The algorithm to reschedule the vessels is implemented by reformulating the optimization model (5)-(18) as a set-partitioning problem by generating all the feasible assignments of the unassigned vessels in the schedule every time there is a disruption. In the optimization run at time instant $t$, the objective function is:

$$
\begin{array}{r}
\min Z_{t}=Z_{1 t}+Z_{2 t}+Z_{3 t} \\
Z_{1 t}=\sum_{p \in P_{u}^{t}}\left(d_{p}^{t} \lambda_{p}+h_{p}^{t}\left(k^{\prime}\right) \lambda_{p}\right) \\
Z_{2 t}=\sum_{p \in P_{u}^{t}}\left(c_{1}\left|b_{p}\left(k^{\prime}\right)-b_{p}(k)\right| \lambda_{p}+c_{2} \mu_{p}\left|e_{p}^{\prime}-e_{p}\right| \lambda_{p}\right) \\
Z_{3 t}=\sum_{p \in P_{o}^{t}}\left(c_{3} w_{p}^{\prime} \lambda_{p}\right)
\end{array}
$$

subject to the constraints

$$
\begin{array}{cc}
\sum_{p}\left(A_{i p} \lambda_{p}\right)=1 & \forall i \in N_{u}^{t} \\
\sum_{p}\left(b_{p}^{k s} \lambda_{p}\right) \leq 1 & \forall k \in M, \forall s \in[t, t+H] \\
\lambda_{p} \in\{0,1\} & \forall p \in P_{u}^{t}
\end{array}
$$

Note that the above model is an extension of the set partitioning model (1)-(4) to solve the deterministic berth allocation problem, including two additional cost terms in the objective function. These two terms, represented by the equations (7)-(8) in the initial optimization model, are related to the minimization of the weighted space and time deviation of the realized schedule from the baseline schedule, and the delays to vessels arriving on-time respectively.

In the above formulation, the following input data is assumed to be available: 


\begin{tabular}{|c|c|}
\hline $\mathrm{H}=$ & et of discrete time intervals in the planning horizon \\
\hline$p^{t}=$ & et of feasible assignments of the unassigned vessels at time instant $t$ \\
\hline$P_{0}^{t}=$ & $\begin{array}{l}P_{u}^{t} \text {, set of feasible assignments of the unassigned vessels at time instant } t \\
\text { at have or are expected to arrive on-time }\end{array}$ \\
\hline $\mathrm{d}_{\mathrm{p}}^{\mathrm{t}}=$ & $\begin{array}{l}\text { rthing delay for the vessel estimated at time instant } t \text { represented by the } \\
\text { signment } p\end{array}$ \\
\hline$h_{p}^{t}\left(k^{\prime}\right)=$ & $\begin{array}{l}\text { andling time of the vessel estimated at time instant } t \text { represented by the } \\
\text { ssignment } p \text { berthed at the starting section } k^{\prime} \in M\end{array}$ \\
\hline$b_{p}(k)=$ & $\begin{array}{l}\text { estimated berthing location as per the baseline schedule of the vessel repre- } \\
\text { sented by the assignment } p\end{array}$ \\
\hline$e_{p}=$ & $\begin{array}{l}\text { he as per the baseline schedule of the vessel repre- } \\
\text { nt } p\end{array}$ \\
\hline$b_{p}\left(k^{\prime}\right)=$ & updated berthing location of the vessel represented by assignment $p$ \\
\hline$e_{p}^{\prime}=$ & updated departure time of the vessel represented by assignment $p$ \\
\hline$w_{p}^{\prime}=$ & $\begin{array}{l}\text { updated time difference between the actual service time and the estimated } \\
\text { service time as per the baseline schedule of the vessel represented by as- } \\
\text { signment } p\end{array}$ \\
\hline$\mu_{\mathrm{p}}=$ & service priority assigned to the vessel represented by the assignment $p$ \\
\hline$c_{1}=$ & cost of shifting the vessel by \\
\hline$c_{2}=$ & $\begin{array}{l}\text { cost of one unit time of delay beyond the departure time of the vessel as per } \\
\text { the baseline schedule }\end{array}$ \\
\hline$c_{3}=$ & $\begin{array}{l}\text { cost of one unit time of additional berthing delay to a vessel arriving on-time } \\
\text { with respect to the baseline schedule }\end{array}$ \\
\hline$A_{\text {ip }}=$ & $\begin{cases}1 & \text { if assignment } p \text { is a feasible assignment for vessel } i \\
0 & \text { otherwise. }\end{cases}$ \\
\hline & $\left\{\begin{array}{l}1 \text { if section } k \text { is occupied at time } s \text { in assignment } p \\
0 \text { otherwise. }\end{array}\right.$ \\
\hline
\end{tabular}

There is only a single type of decision variable used in the model for the selection of the feasible assignments in the optimal solution which is defined as follows:

$$
\lambda_{p}= \begin{cases}1 & \text { if assignment } p \text { is part of the optimal solution; } \\ 0 & \text { otherwise. }\end{cases}
$$

Constraints (23) ensure that each unassigned vessel has exactly one feasible assignment in the optimal solution. Constraints (24) ensure that a given section at a given time can be occupied by at most one vessel. The implementation of the optimization based recovery algorithm is described by Algorithm 2. Note that the berthing assignment of a given vessel may be updated several times during the schedule recovery process, but once the handling of the vessel has actually started, it's berthing assignment does not change again thereafter. 


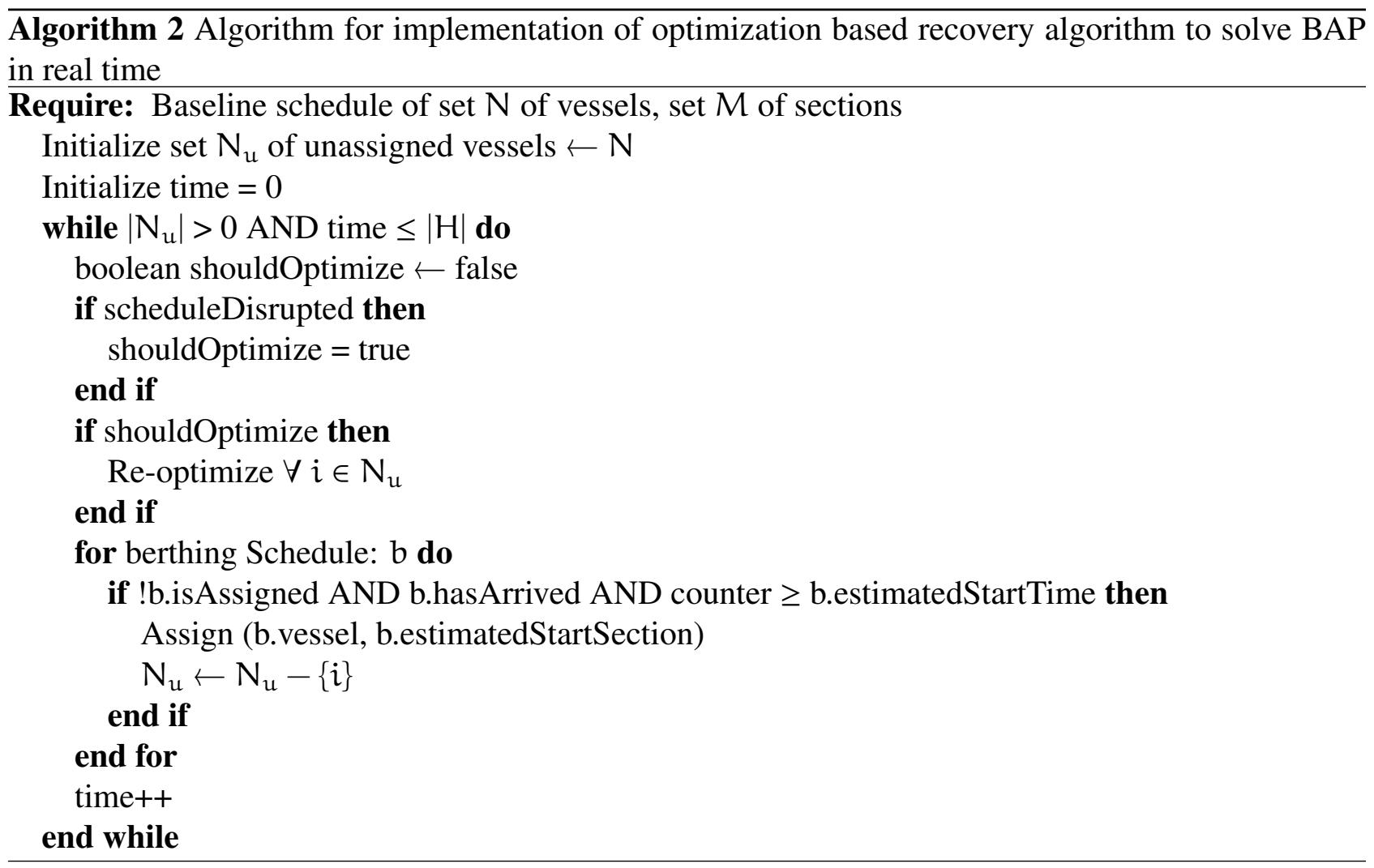

\subsubsection{Smart Greedy Algorithm}

In the smart greedy algorithm, the decision to reschedule a particular vessel is based on the cost of the reassignment of the vessel on the whole schedule by modeling the unknown arrival times and handling times of the vessels as described in section (4.1). In this approach, every time there is an incoming vessel arriving at the port we scan the entire quay and assign it to the set of sections where the total cost of assignment of all the unassigned vessels at that time instant given by equations (5)-( $(8)$ is minimized. As in the traditional greedy method, the assignment of any incoming vessel is done as soon as berthing space is available for the vessel, but not before the planned berthing time as per the original baseline schedule. In determining the total cost to assign a given vessel at a given set of $\operatorname{section}(\mathrm{s})$,

- the arrival times and handling times of all the other unassigned vessels are modeled as described in section (4.1)

- all the other unassigned vessels are assigned to their estimated berthing sections as per the baseline schedule

- the handling of any unassigned vessel cannot start before the estimated berthing time of the vessel as per the original baseline schedule 


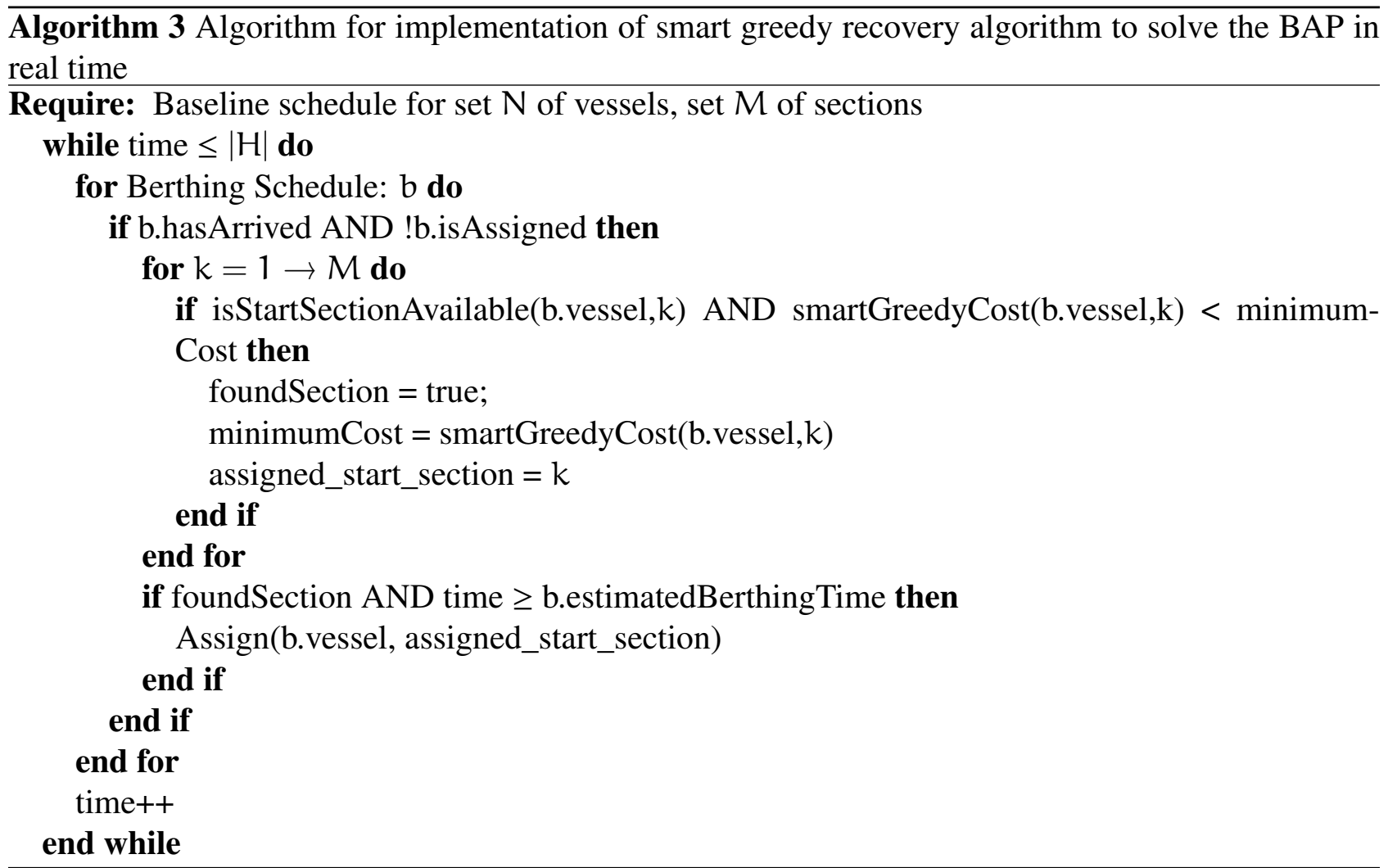

The smart greedy recovery algorithm is described in Algorithm 3. It should be noted that unlike the optimization based recovery method that is based on re-optimization in the event of a disruption and updating the schedule, the smart greedy method is based on reassigning a single vessel at a given time and adhering to the original baseline schedule as far as possible.

\subsubsection{A posteriori Optimization}

If the problem of recovering a planned berthing schedule in real time for a given time horizon is resolved after all the actual arrival and handling time information has been revealed, then the problem of real time recovery reduces to solving the deterministic berth allocation problem with the following objective function cost:

$$
\begin{array}{r}
\min Z_{1}=Z_{1}+Z_{2}+Z_{3} \\
Z_{1}=\sum_{p \in P}\left(d_{p} \lambda_{p}+h_{p}\left(k^{\prime}\right) \lambda_{p}\right) \\
Z_{2}=\sum_{p \in P}\left(c_{1}\left|b_{p}\left(k^{\prime}\right)-b_{p}(k)\right| \lambda_{p}+c_{2} \mu_{p}\left|e_{p}^{\prime}-e_{p}\right| \lambda_{p}\right) \\
Z_{3}=\sum_{p \in P}\left(c_{3} w_{p}^{\prime} \lambda_{p}\right)
\end{array}
$$


subject to the constraints

$$
\begin{array}{cc}
\sum_{p}\left(A_{i p} \lambda_{p}\right)=1 & \forall i \in N \\
\sum_{p}\left(b_{p}^{k s} \lambda_{p}\right) \leq 1 & \forall k \in M, \forall s \in[t, t+H] \\
\lambda_{p} \in\{0,1\} & \forall p \in P
\end{array}
$$

Note that in the above formulation, the index $t$ has been dropped from the variables used in the earlier formulation (19)-(25). A posteriori optimization is useful to test and validate the solution performance of the proposed recovery algorithms, since the solution to the above formulation is a lower bound to the problem of berth rescheduling in real time. Thus it is a used as a benchmark for the comparison of the solution performance of the algorithms.

\section{Results and Analysis}

In this section, we compare the solution performance of the recovery algorithms discussed in the previous section. The algorithms were implemented in JAVA programming language and all tests were run on an Intel Core i7 $(2.80 \mathrm{GHz})$ processor and used a 32-bit version of CPLEX 12.2.

\subsection{Generation of Instances}

In the computational study, the baseline schedule is estimated by solving the deterministic berth allocation problem to optimality, based on instances inspired from real data obtained from SAQR port, Ras Al Khaimah, UAE. The data sample received from the port provided information about the physical attributes of the vessels such as the length and the draft of the vessels, expected and actual times of arrival, berthing times, processing and departure times of vessels, expected and actual berthing positions and the cargo tonnage of the vessels. The data was provided for over 20 vessels for a time horizon of roughly 10 days from 28th March to 6th April, 2011. Based on the data sample and our notes and observations during our visit to the port, we could get an estimate of the range of values for most input parameters in our model.

The relative solution performance of the recovery algorithms is assessed by carrying out a simulation study in which the baseline schedule is subjected to 100 disruption scenarios and the total realized cost of the modified schedule is computed using each recovery method for each simulation run. In our study, the baseline schedule is a combination of cycles of mild or high congestion at the port, as determined by the number of vessels berthing in each cycle. The two baseline schedules considered in the computational study are shown in Figures (4)-(5). The length of each cycle $|\mathrm{H}|$ is equal to 5 days or 120 hours. In a period of mild congestion, the number of scheduled vessel arrivals is 10, while in a period of high congestion it is 25 . As done in practice, the problem of updating the baseline schedule in real time is solved on a rolling planning horizon, where at any given time instant $t$, the planning window from $t$ to $t+H$ is 120 hours. It is further assumed that the port is empty before time $t=0$, 


\begin{tabular}{|c|c|l|c|}
\hline Vessel & ETA & Arrival Updates & ATA \\
\hline Vessel 0 & 19 & 22(2) 21(4) 24(5) 22(6) 24(7) 23(8) 23(9) 23(22) & 23 \\
Vessel 1 & 3 & & 6 \\
Vessel 2 & 4 & $7(3) 6(4) 6(5)$ & 7 \\
Vessel 3 & 14 & $16(2)$ 10(3) 12(4) & 11 \\
Vessel 4 & 18 & $23(9)$ & 22 \\
Vessel 5 & 12 & $13(7)$ & 12 \\
Vessel 6 & 0 & $5(2)$ & 4 \\
Vessel 7 & 0 & & -4 \\
Vessel 8 & 0 & & 3 \\
Vessel 9 & 11 & & 7 \\
\hline
\end{tabular}

Table 4: A sample arrival disruption scenario for $|\mathrm{N}|=10$ vessels. ETA and ATA stand for the expected and actual arrival times respectively. In the arrival updates, the numbers indicate the updated arrival times and the numbers in the parantheses indicate the time instants at which the updates are received.

and the actual arrival times of the incoming vessels are updated at or after this time. A sample arrival disruption scenario is shown in Table 4 .

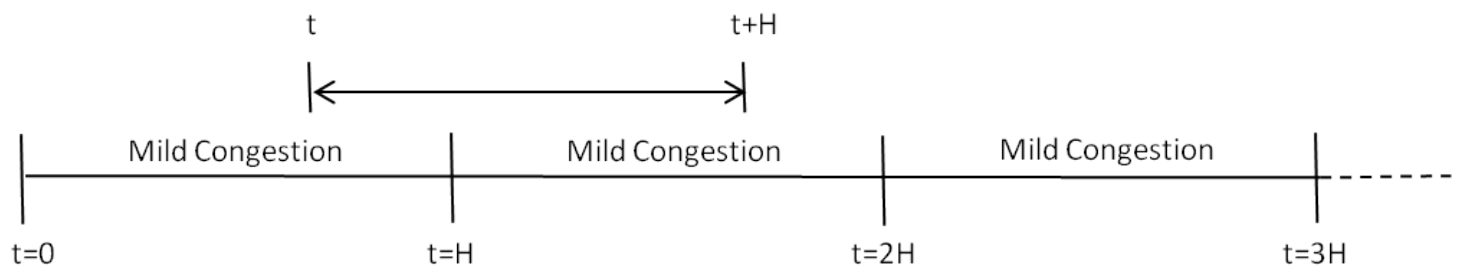

Figure 4: Baseline schedule representing the mildly congested scenario

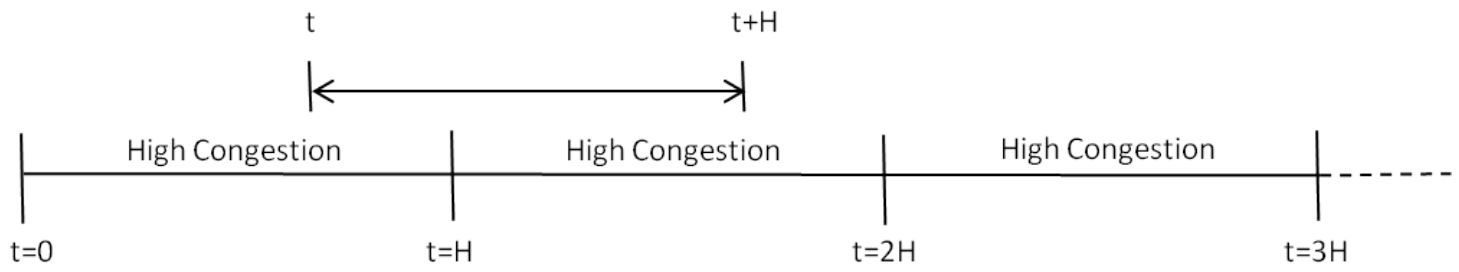

Figure 5: Baseline schedule representing the highly congested scenario

Based on the degree of stochasticity with respect to the deviation in the actual arrival and handling times from the estimated values, two types of disruption scenarios are considered in the simulation study:

- Low Stochasticity

Arrival Time Scenarios: V=5

Handling Time Scenarios: $\gamma=1.1, \tau=0.5$ 
- High Stochasticity

Arrival Time Scenarios: $V=10$

Handling Time Scenarios: $\gamma=1.2, \tau=0.5$

The other parameters of the recovery algorithms are selected on the basis of intuition or by trials and are listed as follows:

- On-time arrival: $\mathrm{U}=4$.

- Uncertainty parameters: $\rho_{\mathrm{a}}=\rho_{\mathrm{h}}=0.95$.

We conduct two sets of computational experiments based on the inclusion or exclusion of the cost component related to the deviation of the realized schedule from the original baseline schedule. The weight constants in the objective function terms $(7)-(8)$ are selected as follows:

- In the first set of experiments, the second cost component $Z_{2 t}$ is neglected, implying that the parameters $c_{1}$ and $c_{2}$ in equation 7 are assumed equal to 0 . The parameter $c_{3}$ in equation 8 is assumed equal to 1 .

- In the second set of experiments, all the three cost omponents are considered. The parameters $c_{1}$ and $c_{3}$ are assumed equal to 1 , while the parameter $c_{2}$ is assumed equal to 0.002 , implying that the cost of shifting a vessel by 500 meters along the quay is considered equivalent to one hour of additional delay.

\subsection{Comparison of Algorithms}

\subsubsection{Excluding Cost of Deviation from the Original Schedule}

In Figures 69, results obtained from the simulation study are shown using box plots for the first set of experiments in which the cost of deviation from the original baseline schedule is neglected in the objective function. It can be seen that the optimization based recovery algorithm and the heuristic based smart greedy recovery method clearly outperform the traditional greedy recovery method for all the four scenarios. Thus if implemented, the proposed algorithms can lead to substanial cost savings to the port.

The optimization based recovery algorithm was found to be better in terms of solution performance than the smart greedy recovery method in most of the tested scenarios. In fact for the mildly congested case, the optimization based method was found to be superior to the other two methods in all the 100 simulation runs. On the other hand, in the high congested case, the performance of the optimization based method was found to be the best in $69 \%$ and $51 \%$ of the simulation runs for the low and high stochasticity scenarios respectively. However note that while the optimization based recovery algorithm outperforms the smart greedy method in terms of solution performance, it is also computationally more expensive as it may take up to a few minutes to run a single re-optimization as 
compared to the smart greedy method where the output is returned almost instantaneously. Moreover since the growth in the number of variables and constraints is very fast in the set-partitioning method, the solver can run out of memory for large instance size as defined by the number of vessels, number of sections along the quay and the length of the planning horizon in the problem instance.

Other key performance indicators to assess the solution performance of the proposed algorithms are shown in the Tables 5.6. The percentage difference in the mean total cost indicates the percentage difference in the mean total objective function cost averaged over the 100 simulations between the recovery algorithm and the solution obtained from the a posteriori optimization method. It can be seen that the solution gap increases with both the level of congestion and the degree of stochasticity. The number of unserved vessels at time $\mathrm{t}=\mathrm{H}$ indicates the count of vessels that are scheduled to arrive between $\mathrm{t}=0$ and $\mathrm{t}=\mathrm{H}$ and have not left the port at time $\mathrm{t}=\mathrm{H}$. Similarly the number of unserved vessels at time $t=2 \mathrm{H}$ indicates the count of vessels that are scheduled to arrive between $t=0$ and $t=2 \mathrm{H}$ and have not left the port at time $t=2 \mathrm{H}$. It can be seen that the optimization based algorithm is superior in terms of both the number of unserved vessels and the average waiting time per vessel. It is interesting to note the significant rise in the average waiting time per vessel expressed in hours with increase in the level of congestion at the port. This result is consistent with our observations during our visit to the port, where the waiting times of few vessels invariably escalate to the order of few days in periods of high congestion and/or stochasticity. The cost of deviation is the objective function cost term in the equation 7 with $c_{1}$ and $c_{2}$ equal to 1 and 0.002 respectively, which is inversely related to the measure of adherence of the realized schedule to the original baseline schedule. Interestingly, in terms of the adherence to the original schedule, the smart greedy method is superior to the other two recovery methods in all the four scenarios. Thus if a key objective of the port is disruption management i.e. minimization of the deviation from the originally planned schedule to minimize the reallocation of resources, the smart greedy method may be the preferred approach to react to disruptions. 
Interquantile range of objective function value

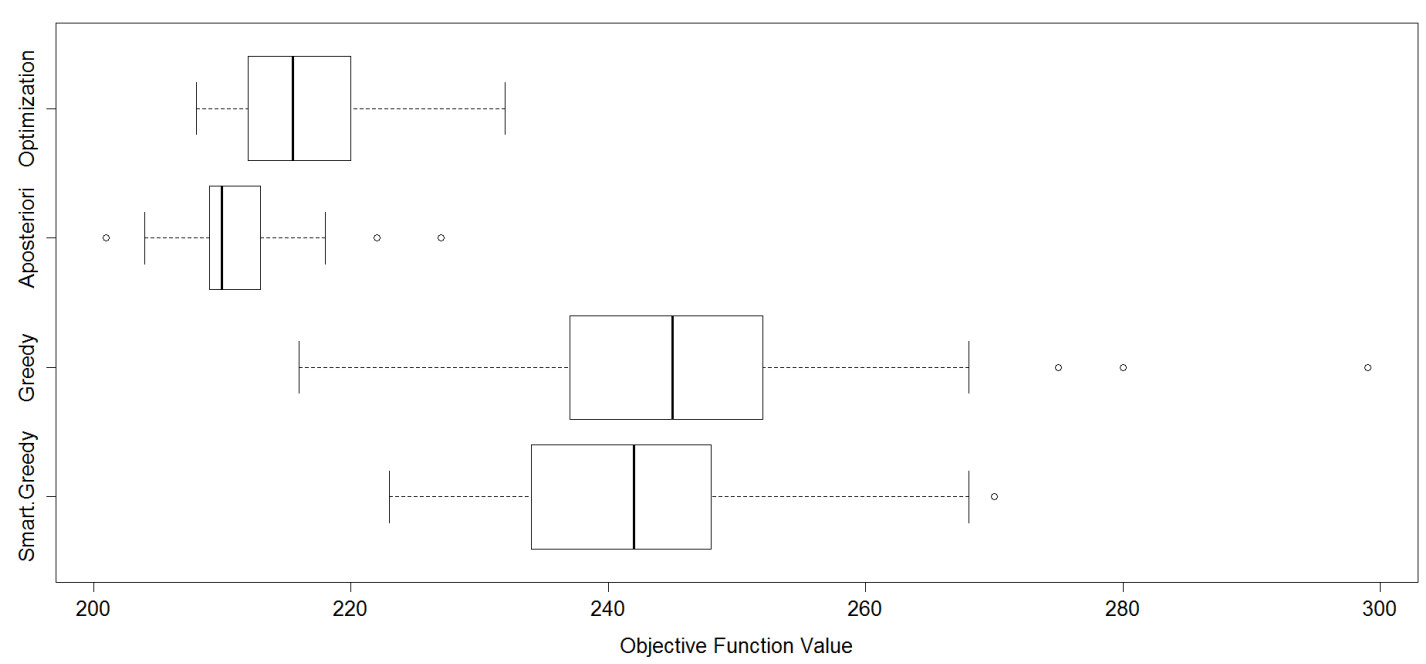

Figure 6: Comparison of the solution performance of the algorithms based on 100 simulation runs for the vessels scheduled to arrive between $\mathrm{t}=\mathrm{H}$ and $\mathrm{t}=2 \mathrm{H}$ for the scenario with mild congestion and low stochasticity

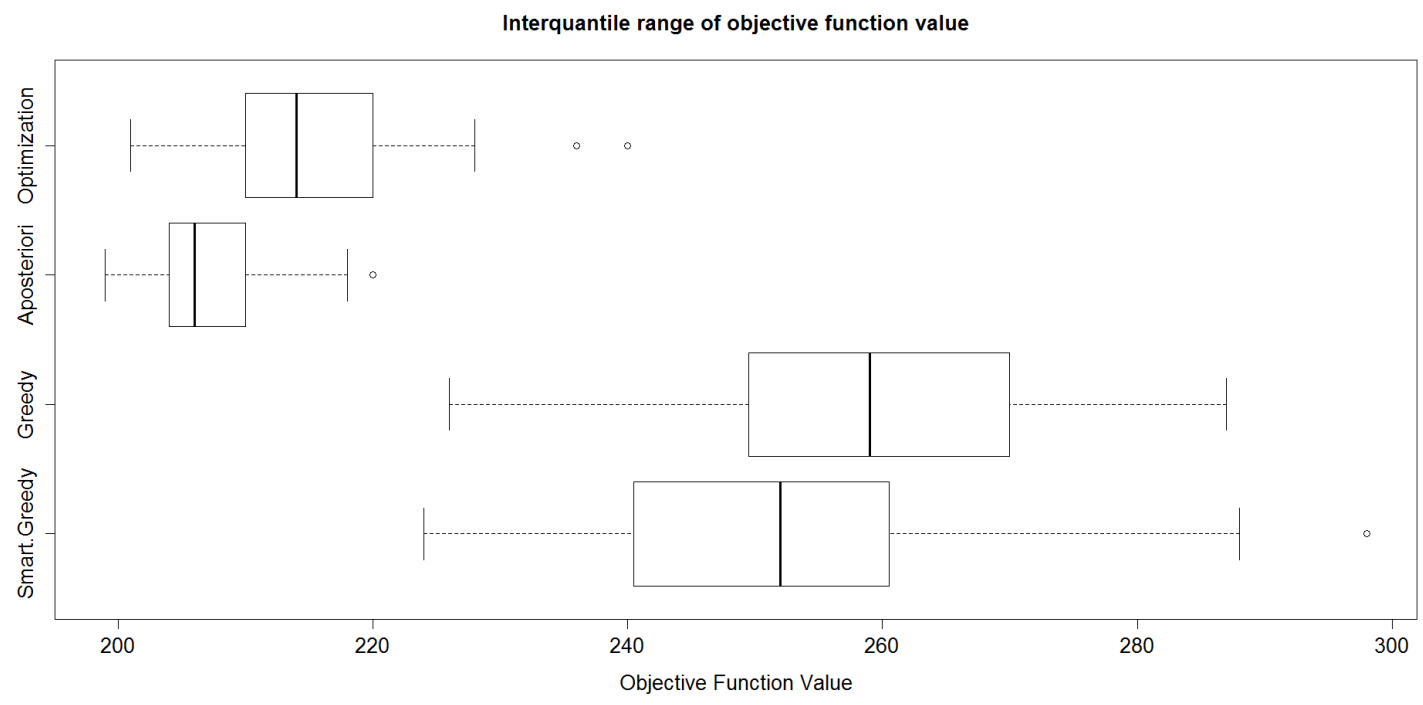

Figure 7: Comparison of the solution performance of the algorithms based on 100 simulation runs for the vessels scheduled to arrive between $\mathrm{t}=\mathrm{H}$ and $\mathrm{t}=2 \mathrm{H}$ for the scenario with mild congestion and high stochasticity 
Interquantile range of objective function value

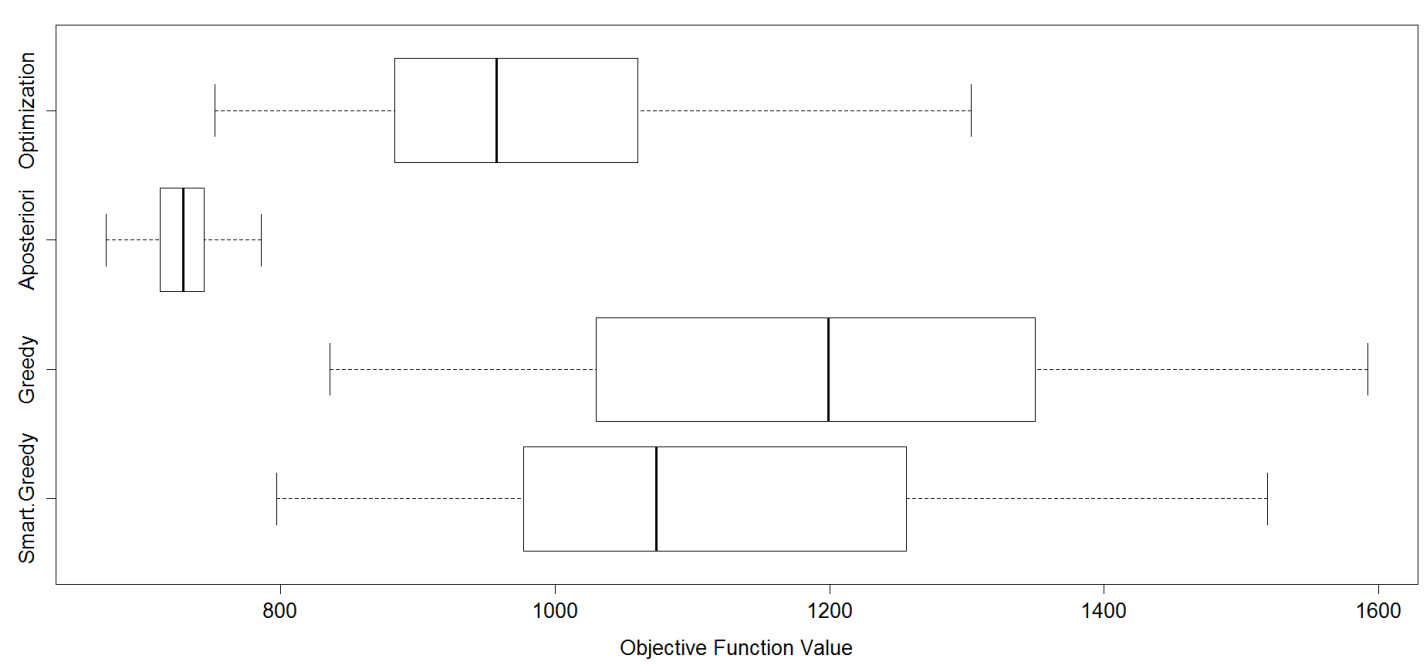

Figure 8: Comparison of the solution performance of the algorithms based on 100 simulation runs for the vessels scheduled to arrive between $\mathrm{t}=\mathrm{H}$ and $\mathrm{t}=2 \mathrm{H}$ for the scenario with high congestion and low stochasticity

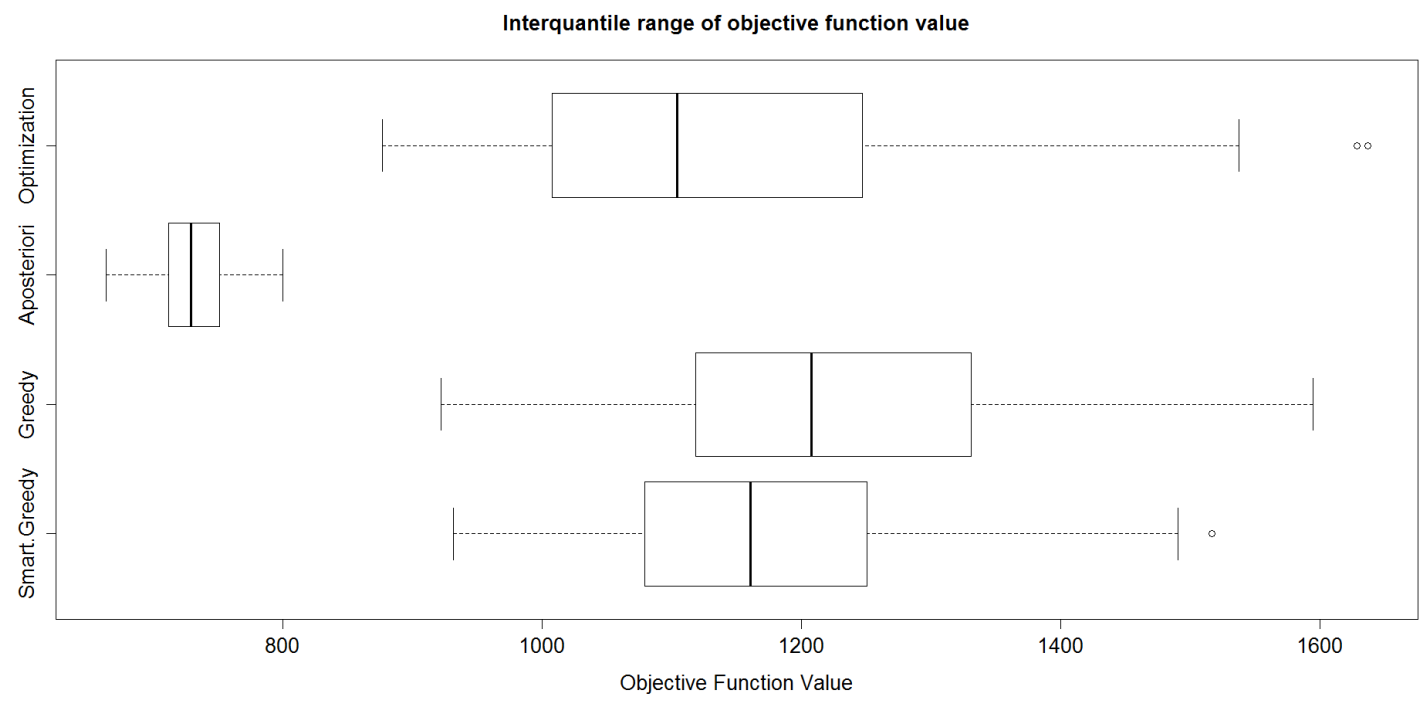

Figure 9: Comparison of the solution performance of the algorithms based on 100 simulation runs for the vessels scheduled to arrive between $\mathrm{t}=\mathrm{H}$ and $\mathrm{t}=2 \mathrm{H}$ for the scenario with high congestion and high stochasticity 


\begin{tabular}{|c|ccc|ccc|}
\hline & \multicolumn{3}{|c|}{ Low Stochasticity } & \multicolumn{3}{c|}{ High Stochasticity } \\
\hline & Optimization & Smart Greedy & Greedy & Optimization & Smart Greedy & Greedy \\
\% difference in mean total cost & $2.77 \%$ & $14.81 \%$ & $16.82 \%$ & $3.75 \%$ & $21.61 \%$ & $25.23 \%$ \\
Number of unserved vessels at $\mathrm{t}=\mathrm{H}$ & 0 & 0 & 0 & 0 & 0 & 0 \\
Number of unserved vessels at $\mathrm{t}=2 \mathrm{H}$ & 0 & 0 & 0 & 0 & 0 & 0 \\
Average waiting time per vessel & 0.12 & 1.43 & 1.67 & 0.32 & 2.72 & 3.03 \\
Average total cost of deviation & 33.96 & 24.77 & 41.99 & 60.404 & 42.78 & 57.42 \\
\hline
\end{tabular}

Table 5: Performance indicators for the mildly congested scenario from $t=H$ to $t=2 \mathrm{H}$ averaged over 100 simulations runs

\begin{tabular}{|c|ccc|ccc|}
\hline & \multicolumn{3}{|c|}{ Low Stochasticity } & \multicolumn{3}{c|}{ High Stochasticity } \\
\hline & Optimization & Smart Greedy & Greedy & Optimization & Smart Greedy & Greedy \\
\% difference in mean total cost & $33.97 \%$ & $51.40 \%$ & $64.45 \%$ & $55.80 \%$ & $61.07 \%$ & $67.29 \%$ \\
Number of unserved vessels at $\mathrm{t}=\mathrm{H}$ & 1.46 & 1.58 & 1.83 & 1.91 & 2.49 & 2.28 \\
Number of unserved vessels at $\mathrm{t}=\mathrm{2H}$ & 1.75 & 2.22 & 2.49 & 2.93 & 3.03 & 3.09 \\
Average waiting time per vessel & 17.10 & 19.67 & 21.45 & 23.02 & 23.40 & 24.62 \\
Average total cost of deviation & 281.66 & 236.3 & 303.52 & 549.64 & 357.83 & 405.48 \\
\hline
\end{tabular}

Table 6: Performance indicators for the highly congested scenario from $t=H$ to $t=2 H$ averaged over 100 simulations runs

\subsubsection{Including Cost of Deviation from the Original Schedule}

In Figures 10, 13, the results are shown when the cost term related to the deviation of the schedule is explicitly considered in the objective function. It can be observed that the proposed optimization based and smart greedy recovery methods significantly outperform the traditional greedy method of reassigning vessels at the port. It can be seen that in general, the solution performance of all the recovery methods deteriorates with increase in the level of congestion and the degree of stochastic variability. The optimization based recovery method outperforms the other recovery methods in all scenarios except the one with high congestion and high stochasticity. Thus it may be inferred that the optimization based recovery method is most sensitive to increase in the level of congestion and degree of stochasticity.

For the mildly congested scenarios, the optimization based recovery method was found to outperform the other two recovery methods in $96 \%$ and $91 \%$ of the simulation runs for the low and high stochasticity scenarios respectively. In the highly congested case on the other hand, the optimization based method returns the best solution in $57 \%$ and $28 \%$ of the simulation runs for the low and high stochasticity scenarios respectively. As can be seen from the Tables 7, 8 , it also does better in terms of the average waiting time and the number of unserved vessels in all except one scenario. In terms of adhering to the original schedule, the superiority of the smart greedy method is established, which performs the best in all but one scenario. 


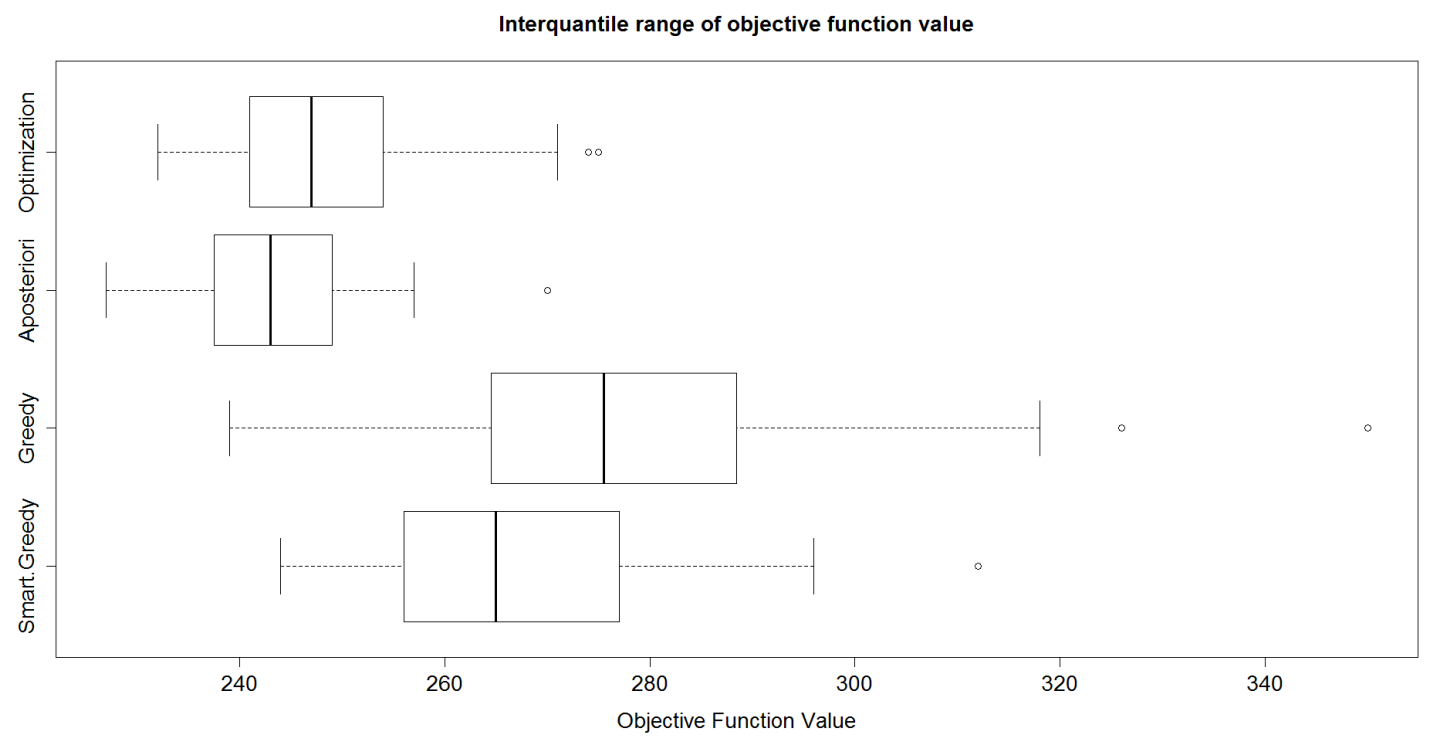

Figure 10: Comparison of the solution performance of the algorithms based on 100 simulation runs for the vessels scheduled to arrive between $\mathrm{t}=\mathrm{H}$ and $\mathrm{t}=2 \mathrm{H}$ for the scenario with mild congestion and low stochasticity

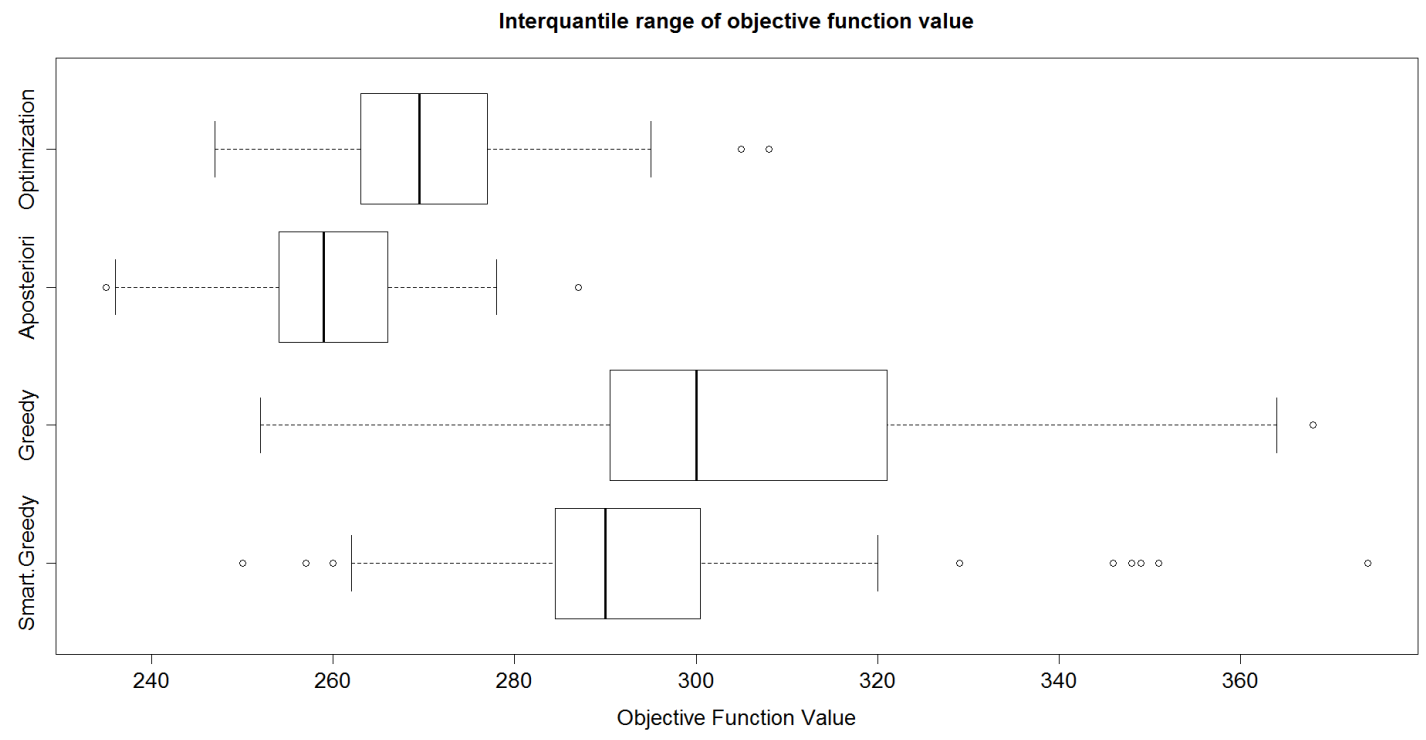

Figure 11: Comparison of the solution performance of the algorithms based on 100 simulation runs for the vessels scheduled to arrive between $\mathrm{t}=\mathrm{H}$ and $\mathrm{t}=2 \mathrm{H}$ for the scenario with mild congestion and high stochasticity 
Interquantile range of objective function value

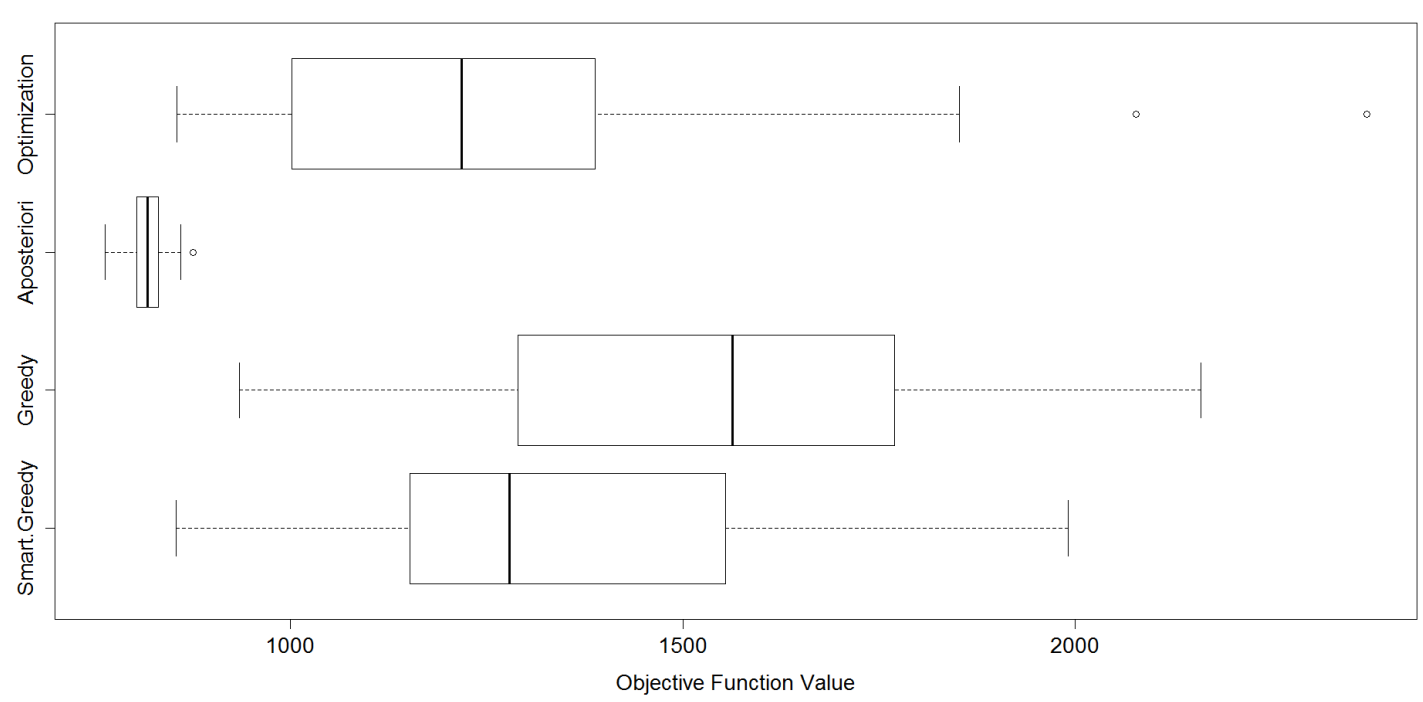

Figure 12: Comparison of the solution performance of the algorithms based on 100 simulation runs for the vessels scheduled to arrive between $\mathrm{t}=\mathrm{H}$ and $\mathrm{t}=2 \mathrm{H}$ for the scenario with high congestion and low stochasticity

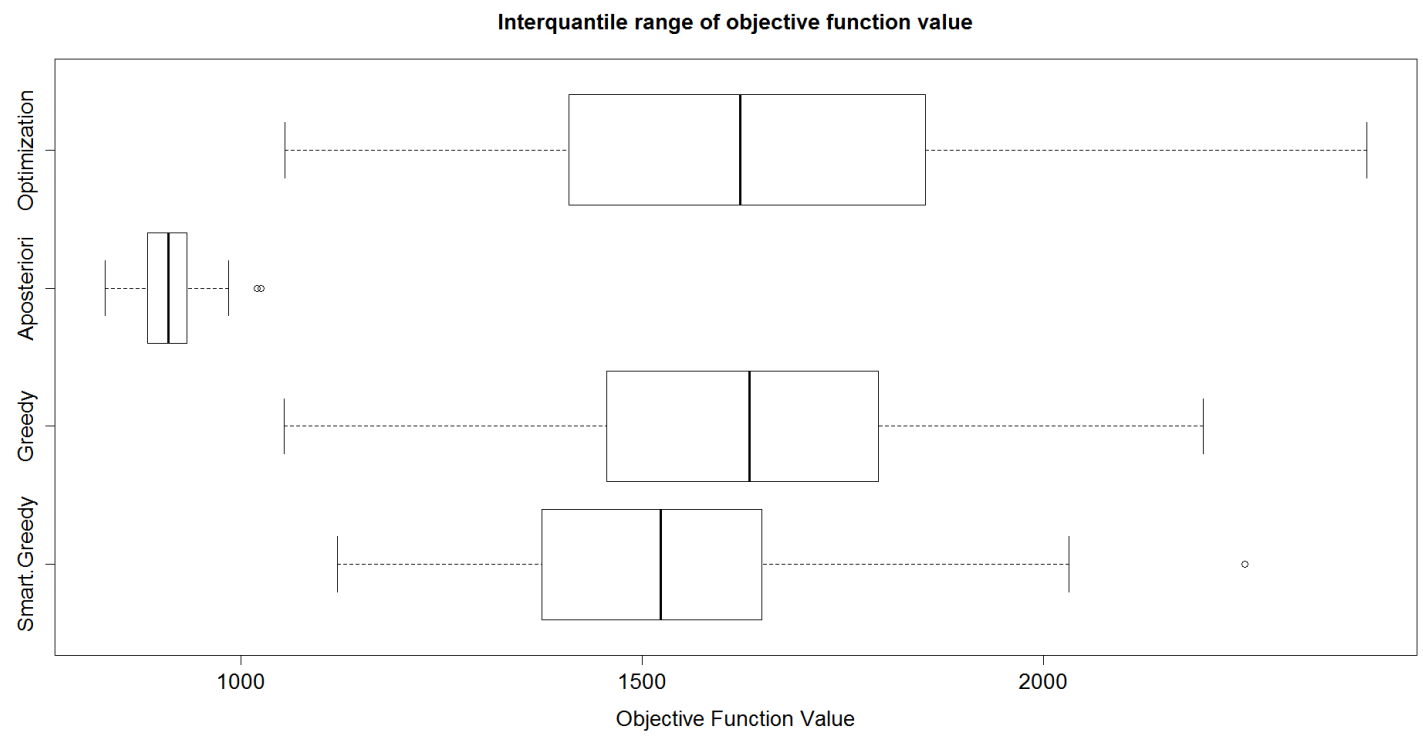

Figure 13: Comparison of the compare the solution performance of the algorithms based on 100 simulation runs for the vessels scheduled to arrive between $\mathrm{t}=\mathrm{H}$ and $\mathrm{t}=2 \mathrm{H}$ for the scenario with high congestion and high stochasticity 


\begin{tabular}{|c|ccc|ccc|}
\hline & \multicolumn{3}{|c|}{ Low Stochasticity } & \multicolumn{3}{c|}{ High Stochasticity } \\
\hline & Optimization & Smart Greedy & Greedy & Optimization & Smart Greedy & Greedy \\
\% difference in mean total cost & $1.78 \%$ & $9.53 \%$ & $13.75 \%$ & $4.11 \%$ & $13.27 \%$ & $17.70 \%$ \\
Number of unserved vessels at $\mathrm{t}=\mathrm{H}$ & 0 & 0 & 0 & 0 & 0 & 0 \\
Number of unserved vessels at $\mathrm{t}=2 \mathrm{H}$ & 0 & 0 & 0 & 0 & 0 & 0 \\
Average waiting time per vessel & 0.283 & 1.426 & 1.5 & 0.636 & 2.681 & 2.863 \\
Average total cost of deviation & 28.87 & 24.38 & 34.97 & 50.79 & 42.3 & 49.3 \\
\hline
\end{tabular}

Table 7: Performance indicators for the mildly congested scenario from $t=H$ to $t=2 \mathrm{H}$ averaged over 100 simulations runs

\begin{tabular}{|c|ccc|ccc|}
\hline & \multicolumn{3}{|c|}{ Low Stochasticity } & \multicolumn{3}{c|}{ High Stochasticity } \\
\hline & Optimization & Smart Greedy & Greedy & Optimization & Smart Greedy & Greedy \\
\% difference in mean total cost & $48.06 \%$ & $63.68 \%$ & $86.85 \%$ & $78.41 \%$ & $68.88 \%$ & $77.57 \%$ \\
Number of unserved vessels at $\mathrm{t}=\mathrm{H}$ & 1.6 & 1.58 & 1.93 & 2.26 & 2.49 & 2.10 \\
Number of unserved vessels at $\mathrm{t}=\mathrm{2H}$ & 1.89 & 2.21 & 2.55 & 3.09 & 2.97 & 3.08 \\
Average waiting time per vessel & 17.85 & 19.65 & 21.82 & 23.60 & 23.34 & 24.38 \\
Average total cost of deviation & 209.1 & 234.74 & 313.11 & 459.25 & 355.53 & 392.88 \\
\hline
\end{tabular}

Table 8: Performance indicators for the highly congested scenario from $t=H$ to $t=2 H$ averaged over 100 simulations runs

\section{Conclusions and Future Work}

In this work, we study and solve the problem of recovering a baseline berthing schedule of vessels at a port in real time as disruptions occur. To the best of our knowledge, very few scholars have attempted to study the problem of real time recovery in port operations, which is typically based on local rescheduling heuristics or simple rules of thumb.

In our study, the underlying model is the dynamic hybrid berth allocation model developed in the context of bulk ports. The uncertainty in the unknown arrival times and handling times of the vessels is modeled based on probability distributions derived from past data. We present an optimization based recovery algorithm based on set partitioning and a heuristic based smart greedy recovery method to reschedule the vessels on a rolling time horizon for a given baseline schedule. The solution performance of the algorithms is tested and validated by conducting a simulation study in which the baseline schedule is the solution of the deterministic berth allocation problem. The results suggest that the proposed methodology for modeling the uncertainty, and the recovery algorithms can significantly reduce the total realized costs of berthing the vessels in comparison to the ongoing practice of re-assigning vessels at the port. The results further indicate that the optimization based method outperforms the other recovery methods in terms of the objective function cost and in terms of some key indicators such as the number of unserved vessels and the average waiting time. However, in terms of the adherence to the originally planned schedule, the smart greedy method is the superior method.

In the future, more work needs to be done to come up with appropriate pricing strategies that can enable the port to earn revenue from the late arriving vessels. Another natural extension of the work done so far is to develop a robust formulation for the berth allocation problem with a certain 
degree of anticipation of delays and variability in information. The recovery algorithms developed in this research can be applied on both the deterministic and robust formulations and the solution performance can be compared to assess the added benefit of robustness. 


\section{References}

Albers, S. (2003). Online algorithms: A survey, Mathematical Programming 97: 3-26. Invited paper at ISMP 2003.

Ben-Tal, A. and Nemirovski, A. (1998). Robust convex optimization, Mathematics of Operations Research 23(4).

Ben-Tal, A. and Nemirovski, A. (1999). Robust solutions of uncertain linear programs, OR Letters 25: $1-13$.

Ben-Tal, A. and Nemirovski, A. (2000). Robust solutions of linear programming problems contaminated with uncertain data, Mathematical Programming 88: 414-424.

Bertsimas, D. and Sim, M. (2003). Robust discrete optimization, Mathematical Programming 98: 4971.

Bertsimas, D. and Sim, M. (2004). The price of robustness, Operations Research 52: 35-53.

Bierwirth, C. and Meisel, F. (2010). A survey of berth allocation and quay crane scheduling problems in container terminals, European Journal of Operational Research 202(3): 615-627.

Birge, J. R. and Louveaux, F. (eds) (1997). Introduction to Stochastic Programming, Springer.

Du, Y., Xu, Y. and Chen, P. (2010). A feedback procedure for robust berth allocation with stochastic vessel delays, Proceedings of the 8th World Congress on Intelligent Control and Automation, Jinan, China.

Han, X.-1., Lu, Z.-q. and Xi, L.-f. (2010). A proactive approach for simultaneous berth and quay crane scheduling problem with stochastic arrival and handling time, European Journal of Operational Research 207(3): 1327 - 1340.

Kall, P. and Mayer, J. (eds) (2005). Stochastic Linear Programming, Models, Theory and Computation, Springer.

Moorthy, R. and Teo, C. P. (2006). Berth management in container terminal: the template design problem, OR Spectrum 28(4): 495-518.

Soyster, A. (1973). Convex programming with set-inclusive constraints and application to inexact linear programming, Operations Research 21(5): 1154-1157.

Stahlbock, R. and Voss, S. (2008). Operations research at container terminals: a literature update, $O R$ Spectrum 30(1): 1-52.

Steenken, D., Voss, S. and Stahlbock, R. (2004). Container terminal operation and operations research - a classification and literature review, OR Spectrum 26(1): 3-49. 
Umang, N., Bierlaire, M. and Vacca, I. (2013). Exact and heuristic methods to solve the berth allocation problem in bulk ports, Transportation Research Part E 54: 14-31.

Wallace, S. and Ziemba, W. (1997). Applications of stochastic programming, (Mps-Siam Series on Optimization), Society for Industrial and Applied Mathematics, Philadelphia, PA, USA.

Xu, Y., Chen, Q. and Quan, X. (2012). Robust berth scheduling with uncertain vessel delay and handling time, Annals of Operations Research 192: 123-140.

Zeng, Q., Yang, Z. and Hu, X. (2012). Disruption recovery model for berth and quay crane scheduling in container terminals, Engineering Optimization 43: 967-983.

Zhen, L. and Chang, D. F. (2012). A bi-objective model for robust berth allocation scheduling, Computers and Industrial Engineering 63: 262-273.

Zhen, L., Lee, L. H. and Chew, E. P. (2011). A decision model for berth allocation under uncertainty, European Journal of Operational Research 212: 54-68. 